\title{
Many-body manifestation of interaction-free measurement: The Elitzur-Vaidman bomb
}

\author{
Oded Zilberberg, ${ }^{1}$ Alessandro Romito, ${ }^{2}$ and Yuval Gefen ${ }^{3}$ \\ ${ }^{1}$ Theoretische Physik, Wolfgang-Pauli-Strasse 27, ETH Zurich, CH-8093 Zurich, Switzerland \\ ${ }^{2}$ Dahlem Center for Complex Quantum Systems and Fachbereich Physik, Freie Universität Berlin, 14195 Berlin, Germany \\ ${ }^{3}$ Department of Condensed Matter Physics, Weizmann Institute of Science, Rehovot, Israel
}

(Received 3 December 2015; revised manuscript received 9 February 2016; published 9 March 2016)

\begin{abstract}
We consider an implementation of the Elitzur-Vaidman bomb experiment in a dc-biased electronic MachZehnder interferometer with a leakage port on one of its arms playing the role of a "lousy bomb." Manybody correlations tend to screen out manifestations of interaction-free measurement. Analyzing the correlations between the current at the interferometer's drains and at the leakage port, we identify the limit where the originally proposed single-particle effect is recovered. Specifically, we find that in the regime of sufficiently diluted injected electron beam and short measurement times, effects of quantum-mechanical wave-particle duality emerge in the cross-current correlations.
\end{abstract}

DOI: 10.1103/PhysRevB.93.115411

\section{INTRODUCTION}

Since its introduction, quantum mechanics has kindled the imagination of scholars due to the interplay of its nonlocal character and particle-wave duality. Using recent technological advances in control over coherent systems, demonstrations of these phenomena are now at the forefront of contemporary research [1]. Of specific interest is the measurement of a quantum particle (described as a wave packet), which unveils its discrete nature when it collapses to reside at a single point. The same particle, before "collapsing," would have assumed a nonlocal character. The compatibility of a particle collapsing at a point and nonlocality has been discussed and demonstrated in the context of the so-called Elitzur-Vaidman (EV) bomb [also known as interaction-free measurement (IFM)]: the wavelike interference of a single quantum particle is modified by the onset of a measurement (bomb) performed at one of the interferometer's arms, which could (but may not) destroy the particle [2].

The interferometer at hand is tuned such that when the "bomb" is absent, wavelike destructive interference renders one of its output ports dark. One then introduces the bomb (hidden in a black box) in one of the interferometer's arms. The bomb implies that even when a particle goes through that arm, it would trigger the bomb, and the setup would explode. If the bomb eventually explodes, one knows a posteriori that the bomb was there. But there is a probability that the bomb does not go off, yet one detects a particle at the interferometer's dark port. That would definitely indicate that the black box has modified the interference pattern, and hence a bomb has been introduced inside the black box. Interestingly, the detection of the presence of the bomb occurs when no interaction with it took place. Notably, there is another possible inconclusive outcome: the bomb does not go off, and the interfering particle exits at the bright port. In that case one does not know whether the bomb was there or not. Additionally, variants of the EVbomb scheme can involve a "lousy" bomb, which explodes with a probability of less than 1 even if the particle passes next to the bomb [3]. Of particular interest to our work is the following observation: no matter how lousy the bomb is, within the many-body context of quantum physics, as the signal in the interferometer is collected over an ensemble of injected particles, there is a vanishing probability that the bomb would remain unexploded at asymptotically long times.

Rather than a bomb, the realization of this EV experimental setup requires the construction of an interferometer with an absorber positioned on one of the interfering paths, as well as the introduction of a single-particle source [4-11]. As such, this topic has remained mostly in the realm of quantum optics, where IFM experiments have been proposed and demonstrated in various systems [4-11] with a variety of applications, including imaging [12], quantum computing [10,13], and single-photon generation [11]. Interestingly, several theoretical studies of the realization and utilization of IFM in electronic solid-state devices were recently pursued by considering, for example, superconducting quantum bits (qubits) [14]. Additionally, an earlier study of electronic Mach-Zehnder interferometers (e-MZIs) $[15,16]$ focused on the employment of a wavelike picture and the influence on the interference signal of a local perturbation in the interferometer. As such, the particle facet of the EV picture was missing. Indeed, e-MZIs are realized using chiral edge modes of quantum Hall bars $[17,18]$, which are one-dimensional channels well described as collective many-body plasmonic waves [19-21]. Typically, these devices are operated at constant voltage bias, leading to the injection of numerous electrons that would eventually, with certainty, trigger the EV bomb. We note, additionally, that single-particle excitations on top of the electron sea in quantum Hall edges have recently been obtained [22]. All this implies that the topic of nonlocality along with wave-particle duality in complex many-electron systems is amenable to experimental studies.

In this paper, we analyze the correlations of transport through an e-MZI with a leaking edge. This is an electronic manifestation of a variant of the EV bomb in which the leaky edge corresponds to an absorber instead of a bomb. In the particlelike limit of this device, the probability of a particle being absorbed and transmitted to the drains at the same time is zero. However, such correlations in the case of many-particles will yield a nonvanishing result. This signifies the fact that the bomb may "explode" even if a signal is detected at the interferometer's dark port. Employing a wavelike scattering matrix formulation, we compute the experimentally measurable many-body correlator and compare it with two limiting 
(a)
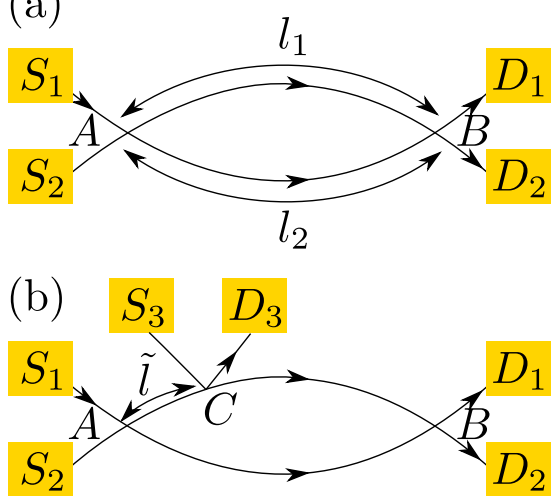

FIG. 1. Illustration of the Mach-Zehnder interferometers (MZIs) under study. Chiral channels are represented by solid lines leading from the sources $\left(S_{1}\right.$, biased, and $S_{2}$, grounded) to the grounded drains $\left(D_{1}\right.$ and $\left.D_{2}\right)$. Interedge tunneling takes place at intersection points. (a) A standard MZI with arms 1 and 2 of lengths $l_{1}$ and $l_{2}$, respectively. (b) The dangling end at $C$ (leading to $D_{3}$ ) serves as an absorber replacing the lousy bomb.

cases (single-particle impinging vs a large influx of particles). Subsequently, we find the conditions for manifesting the wave-particle duality, specifically obtaining the EV physics, in the context of many-body electronic system.

\section{SYSTEM}

We consider a standard e-MZI geometry realized using chiral edge modes of quantum Hall bars [17-21] [see Fig. 1(a)]. Here, particles are injected from the source $S_{1}$ and eventually detected at the drains, $D_{1}$ and $D_{2}$. Note that all channels are chiral; that is, particles may move only in the direction of the arrow and no backscattering occurs.

The evolution of an injected wave packet through the setup is described by considering incoming scattering states from the various sources that are labeled by their quantum number $k$. Schematically, the state of a particle injected from $S_{1}$, after passing through beam splitter $A$ at position $x=0$, is described by $|i\rangle=r_{A}|1\rangle+t_{A}|2\rangle$, where $r_{A}$ and $t_{A}$ are the reflection and transmission amplitudes [23] corresponding to beam splitter $A$ and $|1\rangle,|2\rangle$ are the scattering states corresponding to the upper and lower e-MZI arms. Similarly, beam splitter $B$ is characterized by reflection and transmission amplitudes $r_{B}$ and $t_{B}$, respectively. Between beam splitters $A$ and $B$, orbital phases are accumulated along arm 1 and arm 2, i.e., $e^{i k l_{1}}$ and $e^{i k l_{2}}$, respectively. Additionally, for charged particles in the presence of a magnetic field, the relative phase of the two respective trajectories includes an Aharonov-Bohm phase $\Phi_{\mathrm{AB}} \equiv 2 \pi \frac{\Phi}{\Phi_{0}}$, where $\Phi_{0}$ is a quantum of flux. As the edges are chiral, no higher windings of the accumulated phase are considered. With a proper gauge choice, we reabsorb these phases in an extra phase shift of the transmission coefficient of $t_{B} \rightarrow t_{B} e^{i \phi}$, with the interference phase $\phi=\phi_{B} \equiv k\left(l_{2}-\right.$ $\left.l_{1}\right)+\Phi_{\mathrm{AB}}$.

We incorporate a semitransparent (lousy) absorber on arm 1 of the e-MZI using an additional beam splitter $C$ at position $0<\tilde{l}<l_{1}$ [see Fig. 1(b)]. The propagation of an impinging particle is thus modified: the particle may exit the MZI through arm 3 and reach drain $D_{3}$. The effect of this extra beam splitter evolves the scattering-state component in arm 1, $|1\rangle \rightarrow r_{C}|1\rangle+t_{C}|3\rangle$. This process is commonly referred to as partial collapse and has been studied in the context of qubit uncollapse [24,25] and null weak values [26-28].

This schematic evolution through the e-MZI can be conveniently recast in a scattering matrix formulation; that is, we can write the state of a particle in the interferometer in second quantization, with an annihilation operator

$$
\psi_{k m}(x)=e^{i k x} \begin{cases}a_{k m}, & x<0, \\ b_{k m}, & 0<x<\tilde{l}, \\ c_{k m}, & \tilde{l}<x<l_{2}, \\ d_{k m}, & l_{m}<x .\end{cases}
$$

Here, $m=1,2,3$ labels the different device arms, and we assume arbitrarily that $l_{2}<l_{1}$. The operators $a_{k m}, b_{k m}, c_{k m}, d_{k m}$ are the annihilation operators of momentum eigenstates in the different sectors of the interferometer. They can be arranged in vectors $\mathbf{a}_{k}, \mathbf{b}_{k}, \mathbf{c}_{k}, \mathbf{d}_{k}$, labeled by the arm index $m$, and are related by scattering matrices describing the effects of beam splitters via

$$
\mathbf{b}_{k}=\mathcal{S}_{A} \mathbf{a}_{k}, \mathbf{c}_{k}=\mathcal{S}_{C} \mathbf{b}_{k}, \mathbf{d}_{k}=\mathcal{S}_{B} \mathbf{c}_{k},
$$

with

$$
\begin{aligned}
\mathcal{S}_{i} & =\left(\begin{array}{ccc}
r_{i} & t_{i} & 0 \\
-t_{i}^{*} & r_{i} & 0 \\
0 & 0 & 1
\end{array}\right) \quad i=A, B, \\
\mathcal{S}_{C} & =\left(\begin{array}{ccc}
r_{C} & 0 & -t_{C} \\
0 & 1 & 0 \\
t_{C} & 0 & r_{C}^{*}
\end{array}\right) .
\end{aligned}
$$

\section{SINGLE-PARTICLE LIMIT}

As a first step we analyze the effect of the extra beam splitter $C$ using a schematic single-particle formulation. We assume that the incoming state is labeled by the quantum number $k$, which, for clarity, we omit in the notation below. In the absence of the leakage port, the probability to measure the particle in drain $D_{1}$ is $P_{0}\left(i \rightarrow D_{1}\right)=\left.\left|<D_{1} \| i\right\rangle\right|^{2}$, where $|i\rangle=r_{A}|1\rangle+t_{A}|2\rangle$ includes the effect of beam splitter $A$ and we have defined $\left|D_{1}\right\rangle=r_{B}|1\rangle+t_{B} e^{i \phi}|2\rangle$ to include the effect of beam splitter $B$ and the subsequent detection in $D_{1}$. We have used the subscript ${ }_{0} 0$ to denote the probability in the absence of a leakage port. We obtain for the setup in Fig. 1(a) $P_{0}\left(i \rightarrow D_{1}\right)=\left|r_{A}\right|^{2}\left|r_{B}\right|^{2}+\left|t_{A}\right|^{2}\left|t_{B}\right|^{2}+$ $2\left|r_{A} r_{B} t_{A} t_{B}\right| \cos \left(\phi+\phi_{T}\right)$, where $\phi_{T}=\arg \left(r_{A} r_{B}^{\star} t_{A} t_{B}^{\star}\right)$. We think of the state of the propagating electron as a superposition of qubit states $|1\rangle,|2\rangle$.

Introducing beam splitter $C$ on arm 1 allows state $|1\rangle$ to "leak out" (partial collapse) to branch 3 with probability $\left|t_{C}\right|^{2}$ [see Fig. 1(b)]. The probability to reach drain $D_{3}$ is therefore

$$
P\left(i \rightarrow D_{3}\right)=\left|r_{A}\right|^{2}\left|t_{C}\right|^{2} .
$$

Upon detection of the injected electron in $D_{3}$, we declare the interference experiment void. In such a "partial collapse" state $|1\rangle$ is projected out of the space spanned by $|1\rangle$ and $|2\rangle$. If such 
a projection out does not take place (i.e., the electron is not detected in $D_{3}$ ), the original qubit state is rotated by the measurement's back-action into $\left|i_{C}\right\rangle=(1 / \tilde{\mathcal{N}})\left(r_{A} r_{C}|1\rangle+t_{A}|2\rangle\right)$ with normalization $\tilde{\mathcal{N}}=\sqrt{1-P\left(i \rightarrow D_{3}\right)}$. Consequently, the probability for the particle to subsequently arrive in drain $D_{1}$ is $P\left(i_{C} \rightarrow D_{1}\right) P\left(\overline{i \rightarrow D_{3}}\right)$, where by the overline we denote the complementary event, i.e., $P\left(\overline{i \rightarrow D_{3}}\right)=1-$ $P\left(i \rightarrow D_{3}\right)$. Note that $P\left(i_{C} \rightarrow D_{1}\right)$ can be written using the conditional probability $P\left(i \rightarrow D_{1} \mid \overline{i \rightarrow D_{3}}\right)$. As a result we find that the particle would reach drain $D_{1}$ with the joint probability

$$
\begin{aligned}
P\left(i \rightarrow D_{1}\right)= & P\left(i \rightarrow D_{1}, \overline{i \rightarrow D_{3}}\right)=\left|r_{A}\right|^{2}\left|r_{B}\right|^{2}\left|r_{C}\right|^{2} \\
& +\left|t_{A}\right|^{2}\left|t_{B}\right|^{2}+2\left|r_{C}\right|\left|r_{A} r_{B} t_{A} t_{B}\right| \\
& \times \cos \left(\phi+\phi_{T}+\phi_{C}\right),
\end{aligned}
$$

where $\phi_{C}=\arg \left(r_{C}\right)$. Note that due to causality $P\left(i \rightarrow D_{1}\right)=$ $P\left(i \rightarrow D_{1}, \overline{i \rightarrow D_{3}}\right)$ and, similarly,

$$
P\left(i \rightarrow D_{1}, i \rightarrow D_{3}\right)=0 .
$$

The fact that $P\left(i \rightarrow D_{1}\right) \neq P_{0}\left(i \rightarrow D_{1}\right)$ can be used to detect the presence of the leakage port. Specifically, if the MZI is tuned to have $P_{0}\left(i \rightarrow D_{1}\right)=0$, the detection of a particle at $D_{1}$ in any single realization of the experiment indicates the presence of the leakage port without the particle having leaked out. If the particle is not detected at $D_{1}$, no conclusion about the presence of a leakage channel can be drawn. This is a manifestation of the EV-bomb detection scheme.

It is instructive to recover the results of this single-particle analysis in the scattering matrix formalism, which provides the basis to analyze the statistical many-body effects in the following section. In the scattering matrix formalism we consider the injection of a single particle (in the scattering state $k$ ) into the system, i.e., $\left|i_{k}\right\rangle=a_{k, 1}^{\dagger}|0\rangle$. The detection of the particle in $D_{1(3)}$ is described by the projection operator $\Pi_{D_{1}\left(D_{3}\right)} \equiv d_{k, 1(3)}^{\dagger} d_{k, 1(3)}$. From Eq. (2), the probabilities of the injected particle reaching $D_{1}$ or $D_{3}$ are

$$
\begin{aligned}
& P\left(i_{k} \rightarrow D_{1}\right)=\left\langle 0\left|a_{k, 1} d_{k, 1}^{\dagger} d_{k, 1} a_{k, 1}^{\dagger}\right| 0\right\rangle=\mathcal{A}_{11}, \\
& P\left(i_{k} \rightarrow D_{3}\right)=\left\langle 0\left|a_{k, 1} d_{k, 3}^{\dagger} d_{k, 3} a_{k, 1}^{\dagger}\right| 0\right\rangle=\mathcal{B}_{11},
\end{aligned}
$$

where we have introduced the quantities $\mathcal{A}_{i j} \equiv$ $\left(\mathcal{S}_{A}^{\dagger} \mathcal{S}_{B}^{\dagger} \mathcal{S}_{C}^{\dagger}\right)_{i 1}\left(\mathcal{S}_{C} \mathcal{S}_{B} \mathcal{S}_{A}\right)_{1 j}, \quad \mathcal{B}_{i j} \equiv\left(\mathcal{S}_{A}^{\dagger} \mathcal{S}_{B}^{\dagger} \mathcal{S}_{C}^{\dagger}\right)_{i 3}\left(\mathcal{S}_{C} \mathcal{S}_{B} \mathcal{S}_{A}\right)_{3 j}$. Indeed, an explicit evaluation of $\mathcal{A}_{11}$ and $\mathcal{B}_{11}$ yields for Eqs. (7) and (8) exactly the same expressions as Eqs. (5) and (4), respectively.

Additionally, the joint probability of detecting a particle at $D_{1}$ and $D_{3}$ is given by

$$
\begin{aligned}
P(i & \left.\rightarrow D_{1}, i \rightarrow D_{3}\right)\left\langle 0\left|a_{k, 1} d_{k, 3}^{\dagger} d_{k, 3} d_{k, 1}^{\dagger} d_{k, 1} a_{k, 1}^{\dagger}\right| 0\right\rangle \\
& =\sum_{\beta=1}^{3} \mathcal{A}_{1 \beta} \mathcal{B}_{\beta 1} \equiv 0,
\end{aligned}
$$

where, when the incoming state is that of a single particle, we recover the result in Eq. (6).

The results of this section describe experiments where a single particle is injected into the interferometer. While this is possible in quantum optics, it does not represent the typical experimental conditions of electronic devices. Single-particle sources have been only recently reported in some specifically designed experimental architectures [22]. Since many-electron physics is an essential part of quantum reality, we next analyze this limit.

\section{MANY-BODY CONDITIONAL CORRELATIONS}

In a typical experiment with an e-MZI, particles are injected by applying a constant voltage biased to the source and are collected in the grounded drain over a macroscopically long time. This being the case, only statistical quantities averaged over a many-particle ensemble are accessible, and the signals at the detector correspond to statistical averages of the sourcedrain transition probabilities computed in the previous section. Specifically, for an e-MZI with a constant voltage bias $e V$ at $S_{1}$, the measured current at $D_{1}$ is given by the rate of electrons reaching this drain out of the total rate, $e V / \hbar$, of electrons impinging from the source. The currents through the device are therefore statistical probabilities for an impinging electron to reach the various drains and are precisely given in terms of the probabilities calculated in the single-particle picture above: the current at drain $j$ will be given by $I_{j}=\left(e^{2} / h\right) P\left(i \rightarrow D_{j}\right) V$. When the signal in $D_{1}$ is collected over a large number of particles, any outcome of the IFM experiment would have a macroscopic leakage of particles in $D_{3}$ even if the e-MZI is tuned to have a vanishing current in the absence of the port $D_{3}$. Hence, in the original formulation of the problem with the bomb, the bomb would necessarily explode. In short, under the above conditions the detection of the current at $D_{3}$ does not constitute an uncontested manifestation of IFM.

Can, and, if so, under what conditions, an electronic MZI setup reproduce the original EV bomb measurement scheme? In order to clarify this we focus on the difference between the single-particle results and the many-particle statistical averages relevant for experiments, which appears when dealing with joint probabilities.

This is clearly demonstrated considering, e.g., the statistical joint probability of detecting particles at drains $D_{1}$ and $D_{3}$. In order to relate such a joint probability to a quantity directly accessible in experiments, we next study the constant-current correlations in a many-body (albeit noninteracting) system. We assume that a voltage bias $V$ is applied to the source $S_{1}$, which is held at temperature $T$. For a system with linear dispersion relation, the current operator is $\hat{I}_{i}(x, t)=e v$ : $\psi_{i}^{\dagger}(x, t) \psi_{i}(x, t)$ :, where $\psi_{i}(x, t)$ is the annihilation operator in the $i$ th arm and the normal order operator : · : indicates the subtraction of the mean equilibrium contribution.

We consider the zero-frequency cross-current correlation defined by

$$
F_{1,3} \equiv \frac{h^{2}}{e^{4} V^{4} \tau} \int_{-\tau / 2}^{\tau / 2} d t\left\langle\hat{I}_{3}\left(x_{0}, t\right) \hat{I}_{1}(x, 0)\right\rangle,
$$

where $\tau$ is an infrared cutoff, $\tau \gg \frac{L}{v}$, and $x_{0}, x>l_{1}$. Importantly, since the average current is related to the electron transfer probability by the factor $e^{2} V / h$, the prefactor in the 
definition of $F_{1,3}$ allows us to directly compare this correlator with the averaged joint probability of detecting electrons at drains $D_{1}$ and $D_{3}$ [see Eq. (9)]. Following the derivation detailed in the Appendix, in the limit of $\tau \gg L / v$, Eq. (10) takes the following form:

$$
\begin{aligned}
F_{1,3} \approx & F_{\infty}(\alpha, \Delta \tilde{L})-F_{N}(\alpha, \Delta \tilde{L}) \equiv \frac{1}{\alpha}\left|r_{A}\right|^{2}\left|t_{C}\right|^{2}\left[\alpha\left(\left|t_{A}\right|^{2}\left|t_{B}\right|^{2}+\left|r_{A}\right|^{2}\left|r_{B}\right|^{2}\left|r_{C}\right|^{2}\right)+2 K(\alpha, \Delta \tilde{L})\left|t_{A} t_{B} r_{A} r_{B} r_{C}\right| \cos \left[\Phi\left(\alpha, \Delta \tilde{L}, \Phi_{\mathrm{AB}}\right)\right]\right] \\
& -\frac{1}{\alpha N}\left|r_{A}\right|^{2}\left|t_{C}\right|^{2}\left[L(\alpha)\left(\left|t_{A}\right|^{2}\left|t_{B}\right|^{2}+\left|r_{A}\right|^{2}\left|r_{B}\right|^{2}\left|r_{C}\right|^{2}\right)+2 M(\alpha, \Delta \tilde{L})\left|t_{A} t_{B} r_{A} r_{B} r_{C}\right| \cos \left[\Phi\left(\alpha, \Delta \tilde{L}, \Phi_{\mathrm{AB}}\right)\right]\right],
\end{aligned}
$$

where $F_{\infty}$ and $F_{N}$ are functions of the dimensionless parameters $\quad \alpha=e V \beta /(2 \pi), \quad \Delta \tilde{L}=\pi\left(l_{2}-l_{1}\right) /(\hbar \beta v)$, and $\quad N=e V \tau /(2 \pi \hbar)$. Here, $\beta=1 /\left(k_{B} T\right)$ is the inverse temperature. We have also introduced the functions $K(\alpha, \Delta \tilde{L})=\sin [\alpha \Delta \tilde{L}] / \sinh [\Delta \tilde{L}], \quad L(\alpha)=$ $(\pi \alpha \operatorname{coth}[\pi \alpha]-1) / \pi, M(\alpha, \Delta \tilde{L})=(\pi \sin [\alpha \Delta L] \operatorname{coth}[\pi \alpha]-$ $\Delta \tilde{L} \cos [\alpha \Delta \tilde{L}]) /(\pi \sinh [\Delta L]), \quad$ and $\quad \Phi\left(\alpha, \Delta \tilde{L}, \Phi_{\mathrm{AB}}\right)=$ $\Phi_{\mathrm{AB}}+\phi_{T}+\phi_{C}+\alpha \Delta \tilde{L}$.

Before discussing the implication of this result, it is instructive to contrast the many-body conditional correlator to purely classical correlations of an ensemble of statistically independent impinging electrons. In the latter case, we obtain the statistical average of a joint signal at ports $D_{1}$ and $D_{3}$ :

$$
\begin{aligned}
\tilde{P}\left(i \rightarrow D_{1}, i \rightarrow D_{3}\right)= & P\left(i \rightarrow D_{1}\right) P\left(i \rightarrow D_{3}\right) \\
= & \left|r_{A}\right|^{2}\left|t_{C}\right|^{2}\left[\left|r_{A}\right|^{2}\left|r_{B}\right|^{2}\left|r_{C}\right|^{2}+\left|t_{A}\right|^{2}\left|t_{B}\right|^{2}\right. \\
& \left.+2\left|r_{C}\right|\left|r_{A} r_{B} t_{A} t_{B}\right| \cos \left(\phi+\phi_{T}+\phi_{C}\right)\right] .
\end{aligned}
$$

For a better comparison with the full many-body results that include the effect of averaging over a statistical ensemble due to thermal fluctuations, as well as out-of-equilibrium voltage bias, one can further average over a density matrix $\rho$ that describes an ensemble of initial states. For example, assuming that a voltage bias $V$ is applied to the source $S_{1}$, which is held at temperature $T$, the state of the impinging electrons is described by $\rho=(1 / L) \sum_{k}[f(\hbar v k-e V)-f(\hbar v k)] a_{k 1}^{\dagger} a_{k 1}$, where $f(x)$ is the Fermi-Dirac distribution and the system length $L$ is taken to be the largest length scale in the problem. When averaged over the initial density matrix, the "classical" correlations in Eq. (12) yield

$$
\begin{aligned}
\tilde{P}(i & \left.\rightarrow D_{1}, i \rightarrow D_{3}\right) \\
= & \left|r_{A}\right|^{2}\left|t_{C}\right|^{2}\left[\left|r_{A}\right|^{2}\left|r_{B}\right|^{2}\left|r_{C}\right|^{2}+\left|t_{A}\right|^{2}\left|t_{B}\right|^{2}\right] \\
& +2 \frac{K(\alpha)}{\alpha}\left|r_{C}\right|\left|r_{A} r_{B} t_{A} t_{B}\right| \cos \left[\Phi\left(\alpha, \Delta \tilde{L}, \Phi_{\mathrm{AB}}\right)\right] .
\end{aligned}
$$

Comparing the statistical probability analysis in Eq. (13) with the many-body joint correlation in Eq. (11), we find that $\tilde{P}\left(i \rightarrow D_{1}, i \rightarrow D_{3}\right)=F_{\infty}$, which is the dominant contribution of $F_{1,3}$ when averaging over many electrons, i.e., when $\tau \rightarrow \infty$. Indeed, this represents the well-known fact that $\lim _{\tau \rightarrow \infty} \int_{-\tau / 2}^{\tau_{2}} d t\left\langle\hat{I}_{3}\left(x_{0}, t\right) \hat{I}_{1}(x, 0)\right\rangle=\left\langle\hat{I}_{3}\left(x_{0}, t\right)\right\rangle\left\langle\hat{I}_{1}(x, 0)\right\rangle \tau$. Similarly, a standard analysis of current-current correlations [29] singles out the nontrivial correlations in the crosscurrent noise $S_{1,3} \equiv \lim _{\tau \rightarrow \infty} \int_{-\tau / 2}^{\tau_{2}} d t\left[\left\langle\hat{I}_{3}\left(x_{0}, t\right) \hat{I}_{1}(x, 0)\right\rangle-\right.$ $\left.\left\langle\hat{I}_{3}\left(x_{0}, t\right)\right\rangle\left\langle\hat{I}_{1}(x, 0)\right\rangle\right]$. These nontrivial contributions are encoded in the term $F_{N}=S_{1,3} /\left(\hat{I}_{0}^{2} \tau\right)$ of the many-body cross- current correlation in Eq. (11). Technically, it corresponds to a particle-hole loop contribution and constitutes the quantum corrections to the classical statistical correlations. As we do not include interactions between the injected electrons, this quantum correction generates the single-particle limit sought after in the EV scheme.

While at long averaging times $F_{\infty}$ is the dominant contribution to cross-current correlations, Eq. (11) clearly shows how, for measurements averaged over a finite time, the effects of $F_{\infty}$ and $F_{N}$ are competing. In fact, they become of the same order for short measurement times, such that the average currents are comparable with their fluctuations, i.e., $\left\langle\hat{I}_{3}\left(x_{0}, t\right)\right\rangle\left\langle\hat{I}_{1}(x, 0)\right\rangle \tau \sim$ $S_{1,3}$. In particular, one expects that in the limit where the average number of particles in the interferometer is $\sim 1$ during the measurement time $\tau$, these two terms cancel each other, and we can recover the single-particle result of Eq. (6). By estimating the average number of electrons impinging on the e-MZI during the measurement time by $N=\frac{e V \tau}{2 \pi \hbar}$, we are in the position to interpret the cross-current correlator in terms of a crossover between single-particle quantum-mechanical correlations and classical statistical correlations.

Figure 2(a) depicts the cross-current correlations as a function of the voltage bias $\alpha$ measured in units of temperature for different values of $N$. For any value of $N$, at $\alpha \lesssim 4$, thermal fluctuations dominate over the quantum ones, and the correlations will ultimately reduce to those of classical waves. For large $\alpha$, upon decreasing $N, F_{1,3}$ decreases, and for $N \sim 1$ it is essentially vanishing; that is, we obtain $F_{1,3} \ll F_{\infty}$, which signals that quantum correlations are important. Note that Eq. (11), depicted in Fig. 2, is valid for $\tau \gg L / v$. Recall that as a function of $\alpha$ for a fixed temperature $T, \tau$ changes in order to keep a constant $N$, i.e., $\tau=N \frac{2 \pi \hbar}{e V}=N \frac{\hbar \beta}{\alpha} \sim$ $7.63823 \times 10^{-10}(N / \alpha)$ (seconds), where we considered $T=$ $10 \mathrm{mK}$. Taking experimental values of existing electronic interferometers, $L \sim 10 \mu \mathrm{m}$ and $v \sim 2-6 \times 10^{-4} \mathrm{~m} / \mathrm{s}$ [30], we mark the point $\alpha \equiv N$ as a threshold beyond which our prediction no longer holds. As such, in order to reach the limit of single-particle demonstration of IFM, one should construct smaller interferometers or generate higher-edge mobility. Alternatively, one could consider single-particle injection on top of a Fermi sea [22], but that is beyond the scope of our analysis.

In Fig. 2(b), we see the effect of a finite $\Delta \tilde{L}$. As each wave number experiences a slightly different interference path, both the classical and quantum many-body correlations are affected by averaging over many wave numbers. As a result, when many particles are considered [Eq. (11)], the result moves farther away from the single-particle limit of Eq. (6), reflecting this effective dephasing. Nonetheless, in the limit of short pulses, 


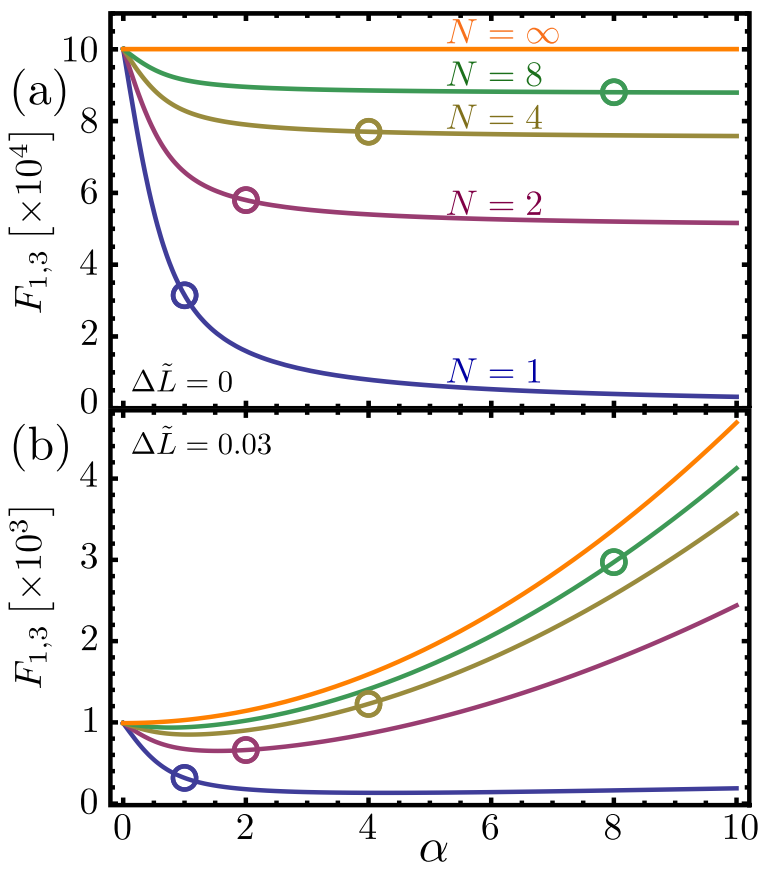

FIG. 2. The many-body cross-current correlator as a function of $\alpha$ [see (11)]. Here, we have taken $\left|t_{C}\right|^{2}=0.3,\left|r_{A}\right|^{2}=\left|r_{B}\right|^{2}=0.5$, and $\Phi_{\mathrm{AB}}+\phi_{T}^{\dagger}+\phi_{C}^{\dagger}=\pi$. For a temperature of $T=10 \mathrm{mK}$ the parameter $\alpha$ corresponds to realistic bias values of up to $\sim 54 \mu \mathrm{V}$. The different plots correspond to different values of $N=1, \ldots, 10$. As a function of $\alpha$ for a fixed $T, \tau$ should be changed in order to keep $N$ constant, i.e., $\tau=N \frac{2 \pi \hbar}{e V}=N \frac{\hbar \beta}{\alpha} \sim 7.63823 \times 10^{-10}(N / \alpha)$ (seconds). We mark by circles the point $\alpha \equiv N$ as the threshold for which our assumption $\tau \gg L / v$ breaks for existing electronic interferometers [30]. (a) The case of $\Delta \tilde{L} \rightarrow 0$. (b) The case of $\Delta \tilde{L}=0.03$, where dephasing affects both the classical and quantum correlators (due to varying interference lengths per wave number). Nonetheless, the single-particle limit remains unaffected, as expected from Eq. (6).

$N=1$, the correlator yields an outcome that agrees with the single-particle picture.

\section{CONCLUSIONS}

The main focus of this study is the assessment of feasible detection of IFM in a genuine many-body electronic system. To this goal, we have analyzed an electronic MZI with a leakage port located on one of the interferometer arms, which serves as an experimentally viable implementation of the EV-bomb gedanken experiment. We considered the typical experimental settings when an ensemble of particles is injected in the interferometer, i.e., the current in the interferometer yields a statistically averaged signal. We analyzed the crosscurrent correlation at the dark and leakage ports, which is vanishing in the single-particle original proposal of the experiment but remains generally finite in the many-particle statistical implementation. This has allowed us to identify the parameters' regime (voltage bias, temperature) for which the many-body correlations approach the single-particle result. We find that the regime where the wave-particle duality emerges lies just at the frontiers of actual experiments with electronic MZIs, where the main limitations are due to the size of the interferometer and the mobility of the electrons at the edges of a Hall bar.

In summary, our results show that the detection of IFM in a many-body electronic system seems to involve two competing facets that need to be dealt with: IFM a la Elitzur-Vaidman requires us to deal with particles (that, in principle, can be pinpointed to a specific spatial coordinate); at the same time, the setup employed is an interferometer, which invokes the wave character of the quantum object. One thus needs to finetune the system to zoom in on a regime where particle-wave duality is manifest. Our analysis might trigger experiments with single-electron biased MZIs, where this physics may be elucidated.

\section{ACKNOWLEDGMENTS}

This work has been supported by the Swiss National Science Foundation, the German-Israel Foundation (GIF), Deutsche Forschungsgemeinschaft (DFG) Grants No. RO 2247/8-1 and No. RO 4710/1-1, and the Israel Science Foundation (ISF).

\section{APPENDIX: DERIVATION OF THE MANY-BODY CORRELATOR}

In this Appendix, we provide additional details of the derivation of Eq. (11). We start with the definition of the cross-current correlator (10). Plugging in the definition of the current operators, we have an expression of the form

$$
\begin{aligned}
F_{1,3}= & \frac{h^{2}}{e^{4} V^{4} \tau} \int_{-\tau / 2}^{\tau / 2} d t\left\langle: \psi_{3}^{\dagger}\left(x_{0}, t\right) \psi_{3}\left(x_{0}, t\right)\right. \\
& \left.\times:: \psi_{1}^{\dagger}(x, 0) \psi_{1}(x, 0):\right\rangle .
\end{aligned}
$$

Using Wick's theorem and writing the fermion operators in momentum space [see Eq. (1)], we obtain

$$
\begin{aligned}
F_{1,3}= & \frac{h^{2}}{e^{4} V^{4} L^{2} \tau} \int_{-\tau / 2}^{\tau / 2} d t \sum_{k_{1}, k_{2}, k_{3}, k_{4}} e^{i x\left(k_{1}-k_{3}\right)+i x_{0}\left(k_{2}-k_{4}\right)} \\
& \times\left\{\left\langle d_{k_{4} 3}^{\dagger}(t) d_{k_{1} 1}(0)\right\rangle\left\langle d_{k_{2} 3}(t) d_{k_{3} 1}^{\dagger}(0)\right\rangle\right. \\
& \left.\left.+\left\langle: d_{k_{4} 3}^{\dagger}(t) d_{k_{2} 3}(t):\right\rangle: d_{k_{3} 1}^{\dagger}(0) d_{k_{1} 1}(0):\right\rangle\right\}
\end{aligned}
$$

Similar to what we have performed in the calculation of Eqs. (7) and (8), we introduce the scattering matrix definitions:

$$
\begin{aligned}
F_{1,3}= & \frac{h^{2}}{e^{4} V^{4} L^{2} \tau} \int_{-\tau / 2}^{\tau / 2} d t \sum_{k_{1}, k_{2}, k_{3}, k_{4}} e^{i x\left(k_{1}-k_{3}\right)+i x_{0}\left(k_{2}-k_{4}\right)} \\
& \times \sum_{\alpha, \beta, \gamma, \delta=1}^{3}\left[\mathcal{A}_{\alpha \beta} \mathcal{B}_{\gamma \delta}\right]\left(k_{1}, \ldots, k_{4}\right) \\
& \times\left\{\left\langle a_{k_{4} \alpha}^{\dagger}(t) a_{k_{1} \delta}(0)\right\rangle\left\langle a_{k_{2} \beta}(t) a_{k_{3} \gamma}^{\dagger}(0)\right\rangle\right. \\
& \left.\left.+\left\langle: a_{k_{4} \alpha}^{\dagger}(t) a_{k_{2} \beta}(t):\right\rangle: a_{k_{3} \gamma}^{\dagger}(0) a_{k_{1} \delta}(0):\right\rangle\right\}
\end{aligned}
$$

The fermion operators are diagonal in momentum and are uncorrelated between the edges, i.e., $\left\langle a_{\alpha, k^{\prime}}^{\dagger}(t) a_{\beta, k}(t)\right\rangle=$ $\left\langle a_{\alpha, k}^{\dagger}(t) a_{\alpha, k}(t)\right\rangle \delta_{\alpha, \beta} \delta_{k, k^{\prime}}$. We additionally can write the 
free-fermion time dependence, e.g., $a(t)=e^{-i v t}$, and obtain

$$
\begin{aligned}
F_{1,3}= & \frac{h^{2}}{e^{4} V^{4} L^{2} \tau} \int_{-\tau / 2}^{\tau / 2} d t \sum_{k_{1}, k_{2}} \sum_{\alpha, \beta=1}^{3}\left\{e^{i\left(k_{1}-k_{2}\right)\left[\left(x-x_{0}\right)+v t\right]}\left[\mathcal{A}_{\alpha \beta} \mathcal{B}_{\beta \alpha}\right]\left(k_{1}, k_{2}\right)\left\langle a_{k_{1} \alpha}^{\dagger} a_{k_{1} \alpha}\right\rangle\left\langle a_{k_{2} \beta} a_{k_{2} \beta}^{\dagger}\right\rangle\right. \\
& \left.\left.+\mathcal{A}_{\alpha \alpha}\left(k_{1}\right) \mathcal{B}_{\beta \beta}\left(k_{2}\right)\left\langle: a_{k_{1} \alpha}^{\dagger} a_{k_{1} \alpha}:\right\rangle: a_{k_{2} \beta}^{\dagger} a_{k_{2} \beta}:\right\rangle\right\} .
\end{aligned}
$$

Performing the time integral and writing in the Fermi-Dirac distribution, $f_{i}(k) \equiv\left\langle a_{k i}^{\dagger} a_{k i}\right\rangle=f\left(\hbar v k-\mu_{i}\right)=\frac{1}{e^{\beta\left(\hbar v k-\mu_{i}\right)}+1}$, we reach

$$
\begin{aligned}
F_{1,3}= & \frac{h^{2}}{e^{4} v V^{4} L^{2} \tau} \sum_{k_{1}, k_{2}} \sum_{\alpha, \beta=1}^{3}\left\{\frac{2 \sin \left[\left(k_{1}-k_{2}\right) v \tau / 2\right]}{\left(k_{1}-k_{2}\right)} e^{i\left(k_{1}-k_{2}\right)\left(x-x_{0}\right)}\left[\mathcal{A}_{\alpha \beta} \mathcal{B}_{\beta \alpha}\right]\left(k_{1}, k_{2}\right) f_{\alpha}\left(k_{1}\right)\left[1-f_{\beta}\left(k_{2}\right)\right]\right. \\
& \left.+\tau v \mathcal{A}_{\alpha \alpha}\left(k_{1}\right) \mathcal{B}_{\beta \beta}\left(k_{2}\right)\left[f_{e V}\left(k_{1}\right)-f_{\alpha}\left(k_{1}\right)\right]\left[f_{\beta}\left(k_{2}\right)-f_{0}\left(k_{2}\right)\right]\right\},
\end{aligned}
$$

where we have used the chemical potential configuration of our device, $f_{2}(k)=f_{3}(k) \equiv f_{0}(k) \equiv f(\hbar v k) \equiv\left\langle a_{k i}^{\dagger} a_{k i}\right\rangle_{0}$ and $f_{1}(k) \equiv$ $f_{e V}(k) \equiv f(\hbar v k-e V)$. Taking the limit $\tau \gg L / v$, we can perform the $k_{2}$ sum in the first expression and obtain

$$
\begin{aligned}
F_{1,3} \approx & \frac{h^{2}}{e^{4} v V^{4} L \tau}\left[\sum_{\alpha, \beta=1}^{3} \sum_{k} \mathcal{A}_{\alpha \beta}(k) \mathcal{B}_{\beta \alpha}(k) f_{\alpha}(k)\left[1-f_{\beta}(k)\right]\right] \\
& +\frac{h^{2}}{e^{4} V^{4} L^{2}} \sum_{\alpha=2}^{3} \sum_{k, k^{\prime}}\left\{\mathcal{B}_{11}(k)\left[f_{1}(k)-f_{0}(k)\right] \mathcal{A}_{\alpha \alpha}\left(k^{\prime}\right)\left[f_{e V}\left(k^{\prime}\right)-f_{\alpha}\left(k^{\prime}\right)\right]\right\} .
\end{aligned}
$$

Using the identity $f_{\alpha}\left(1-f_{\beta}\right)=\frac{1}{2}\left[f_{\alpha}\left(1-f_{\alpha}\right)+f_{\beta}\left(1-f_{\beta}\right)+\left(f_{\alpha}-f_{\beta}\right)+\left(f_{\alpha}-f_{\beta}\right)^{2}\right]$ and the explicit definitions of the scattering matrix elements, we arrive at

$$
\begin{aligned}
F_{1,3} \approx & -\frac{h^{2}}{e^{4} v V^{4} L \tau}\left[\sum_{k}\left|r_{A}\right|^{2}\left|t_{C}\right|^{2} \mathcal{B}_{11}(k)\left[f_{e V}(k)-f_{0}(k)\right]^{2}\right] \\
& +\frac{h^{2}}{e^{4} V^{4} L^{2}} \sum_{k, k^{\prime}}\left\{\left(1-\left|r_{A}\right|^{2}\left|t_{C}\right|^{2}\right) \mathcal{B}_{11}(k)\left[f_{e V}(k)-f_{0}(k)\right]\left[f_{e V}\left(k^{\prime}\right)-f_{\alpha}\left(k^{\prime}\right)\right]\right\} .
\end{aligned}
$$

The summation over momenta $k$ and $k^{\prime}$ is replaced by energy integrals, $1 / L \sum_{k} \rightarrow 1 /(2 \pi \hbar v) \int d E$, and expression $(11)$ is readily obtained.

[1] S. Haroche, Rev. Mod. Phys. 85, 1083 (2013).

[2] A. C. Elitzur and L. Vaidman, Foundations of Physics 23, 987 (1993).

[3] G. Mitchison and S. Massar, Phys. Rev. A 63, 032105 (2001).

[4] P. Kwiat, H. Weinfurter, T. Herzog, A. Zeilinger, and M. A. Kasevich, Phys. Rev. Lett. 74, 4763 (1995).

[5] E. H. du Marchie van Voorthuysen, Am. J. Phys. 64, 1504 (1996).

[6] M. Hafner and J. Summhammer, Phys. Lett. A 235, 563 (1997).

[7] T. Tsegaye, E. Goobar, A. Karlsson, G. Björk, M. Y. Loh, and K. H. Lim, Phys. Rev. A 57, 3987 (1998).

[8] P. G. Kwiat, A. G. White, J. R. Mitchell, O. Nairz, G. Weihs, H. Weinfurter, and A. Zeilinger, Phys. Rev. Lett. 83, 4725 (1999).

[9] J.-S. Jang, Phys. Rev. A 59, 2322 (1999).

[10] O. Hosten, M. T. Rakher, J. T. Barreiro, N. A. Peters, and P. G. Kwiat, Nature (London) 439, 949 (2006).

[11] F. Wolfgramm, Y. A. de Icaza Astiz, F. A. Beduini, A. Cerè, and M. W. Mitchell, Phys. Rev. Lett. 106, 053602 (2011).

[12] A. G. White, J. R. Mitchell, O. Nairz, and P. G. Kwiat, Phys. Rev. A 58, 605 (1998).

[13] L. Vaidman, Phys. Rev. Lett. 98, 160403 (2007).
[14] G. S. Paraoanu, Phys. Rev. Lett. 97, 180406 (2006).

[15] E. Strambini, L. Chirolli, V. Giovannetti, F. Taddei, R. Fazio, V. Piazza, and F. Beltram, Phys. Rev. Lett. 104, 170403 (2010).

[16] L. Chirolli, E. Strambini, V. Giovannetti, F. Taddei, V. Piazza, R. Fazio, F. Beltram, and G. Burkard, Phys. Rev. B 82, 045403 (2010).

[17] B. I. Halperin, Phys. Rev. B 25, 2185 (1982).

[18] X. G. Wen, Phys. Rev. B 41, 12838 (1990).

[19] J. von Delft and H. Schoeller, Ann. Phys. (Berlin, Ger.) 7, 225 (1998).

[20] C. Texier and M. Büttiker, Phys. Rev. B 62, 7454 (2000).

[21] Y. Ji, Y. Chung, D. Sprinzak, M. Heiblum, D. Mahalu, and H. Shtrikman, Nature (London) 422, 415 (2003).

[22] J. Dubois, T. Jullien, F. Portier, P. Roche, A. Cavanna, Y. Jin, W. Wegscheider, P. Roulleau, and D. Glattli, Nature (London) 502, 659 (2013).

[23] We assume here and throughout the paper that the scattering matrix elements are independent of $k$.

[24] L. P. Pryadko and A. N. Korotkov, Phys. Rev. B 76, 100503 (2007). 
[25] N. Katz, M. Neeley, M. Ansmann, R. C. Bialczak, M. Hofheinz, E. Lucero, A. O'Connell, H. Wang, A. N. Cleland, J. M. Martinis et al., Phys. Rev. Lett. 101, 200401 (2008).

[26] O. Zilberberg, A. Romito, D. J. Starling, G. A. Howland, C. J. Broadbent, J. C. Howell, and Y. Gefen, Phys. Rev. Lett. 110, 170405 (2013).
[27] O. Zilberberg, A. Romito, and Y. Gefen, Phys. Scr. T151, 014014 (2012).

[28] O. Zilberberg, A. Romito, and Y. Gefen, in Quantum Theory: A Two-Time Success Story (Springer, Berlin, 2014), pp. 377-387.

[29] Y. M. Blanter and M. Buttiker, Phys. Rep. 336, 1 (2000).

[30] I. Neder, N. Ofek, Y. Chung, M. Heiblum, D. Mahalu, and V. Umansky, Nature (London) 448, 333 (2007). 